\title{
Sublayer composition evaluation of Artwork using active thermography
}

\author{
by G. Steenackers***, J. Peeters*, K. Janssens*** \\ * University of Antwerp, Op3Mech research group, Groenenborgerlaan 171, B-2020 Antwerp, Belgium, \\ gunther.steenackers@uantwerpen.be \\ ${ }_{* *}$ Vrije Universiteit Brussel (VUB), Acoustics \& Vibration Research Group (AVRG), B-1000 Brussels, Belgium \\ *** University of Antwerp, AXES research group, Groenenborgerlaan 171, B-2020 Antwerp, Belgium
}

\begin{abstract}
Infrared artwork inspection is typically performed through active thermography and infrared reflectography (IRR) with different setups and cameras. While IRR is an established technique in the museum field, exploiting mainly the IR-A $(0.7-1.4 \mu \mathrm{m})$ band to probe for hidden layers and modifications within the paint stratigraphy system, active thermography operating in the IR-C range ( $3-5 \mu \mathrm{m})$ is less frequently employed with the aim to visualize structural defects and features deeper inside the build-up. In this work, we investigate the usability of an IR-B+C system to identify overpainted works of art below a relatively thick absorbing layer of lead white paint.
\end{abstract}

Keywords: Active thermography; Artwork inspection; IR reflectography

\section{Introduction}

Thermography has many advantages including operating in a non-contact mode, it can be performed in situ, it can cover large areas and it is a quantitative method [1]. The disadvantages are that anomalies deeper in the structure are more difficult to inspect [2] [3] and that the results are highly dependent on the used experimental set-up conditions, for example emissivity variations and non-homogeneous surface heating. Efficient signal processing and filtering of the anomaly information in the field of active thermography methods are very important. With the help of adapted image- and signal processing algorithms, it is possible to detect small discontinuities inside structures or extract material characteristic information.

The most important artworks (i.e., canvas paintings, mural paintings, panel paintings, inlay works, icons) can be considered as multi-layer structures, in which each layer fulfils a key role. Therefore, important proofs linked to the history of the artefact may be partially lost in time. At this point, the use of techniques which improve the readability of artefacts undetectable to the naked eye is highly desirable.

Using radiation beyond the visible range, different characteristics of the inspected artwork may be revealed according to the bandwidth acquired. Beyond the traditional diagnostic methods, such as reflectography, thermography, selective multi-spectral analysis in the near-infrared region IR-A $(0.7-1.4 \mu \mathrm{m})$ has recently been demonstrated to be a promising tool for investigating pictorial layers [1],[2],[6]. In [7], the authors applied high resolution IR-A reflectography and IR-C' $(3-5 \mu \mathrm{m})$ imaging to characterize the superficial layer of a fresco and to analyse the stratigraphy of different pictorial layers. IR-C" $(7-14 \mu \mathrm{m})$ thermography was also used to understand the nature of the support.

The non-invasive methods near-infrared (IR-A), short-wave infrared (IR-A) and thermographic inspection (IR-C) of artworks were described in [8] and also compared in terms of their ability to reveal both hidden graphite containing underdrawings and subsurface degradations. Instead, the performance of transient thermography, three optical methods and ultrasonic testing applied together on a veneered wood sample with real and fabricated defects were explored in [9].

\section{Methodology}

Within this work, a comparison is made between IRR reflectography and active thermography with respect to the identification of a sublayer composition or a so called painting below painting. Therefore the results of a Xenics Xeva camera with spectral range $0.9-1.7 \mu \mathrm{m}$ and spatial resolution of $640 \times 512$ and a $100 \mathrm{~W}$ incandescent lamp with carbon filament as illumination source is used for the IRR Reflectography measurements. The active thermography results are retrieved using a FLIR X6540sc camera, sensitive in the $3-5 \mu \mathrm{m}$ spectral band with a spectral resolution of $640 \times 512$ pixels and NETD of $18 \mathrm{mK}$. The active controlled excitation unit consists of a Xenon flash unit of $6.4 \mathrm{~kJ}$.

A replica of a Mondrian painting covered with a thick lead white layer and on top of it a different Mondrian replica is used as test sample. The goal of this sample is to find traces of the replica underneath the lead white layer. The overview of both replicas is shown in Fout! Verwijzingsbron niet gevonden. and the full composition is schematically provided in Fout! Verwijzingsbron niet gevonden..

Infrared reflectography is well-established for art work inspection, in order to reveal pentimenti, touch-ups and other artefacts [8]. It is a photographic technique at a longer wavelength, which makes it feasible to use the transparency of most of the pigments in this spectral range to evaluate the layers underneath optical opaque layers. The technique is 
mostly performed in the IR-A spectrum, but it is possible in the IR-B spectrum as well. The degree of paint opacity is mostly determined by two factors: the absorption of light by the pigment and the scattering of light by pigment particles. A full description can be found in the work of Gavrilov et al.[10]. Within the measurement set-up, a halogen float lamp of 1000 Wor IR LED $940 \mathrm{~nm}$ with a power of $800 \mathrm{~W}$ is made use of as an illumination source. The acquired measurement data is not further post-processed before interpretation.

Active thermography uses the transient behaviour of the response on a thermal excitation (heating or cooling) of the object to identify anomalies within the structure as shown in Figure 1. The study of active thermography has become an important Non-Destructive Evaluation technique (NDE) for damage detection and material updating in metallic structural components [11], as well as CFRP (Carbon Fiber Reinforced Polymer) and GFRP (Glass Fiber Reinforced Polymer) composites [12] In state-of-the-art research, the technique is upcoming for the inspection of art works in the IR-C spectrum [8].

The illumination and excitation sources for all test samples are positioned in front of the test sample on a distance of $1 \mathrm{~m}$, except when further specified. An overview of the discussed measurements is shown in Table 1.

Table 1. Overview of the different experiments used within this study in order of appearance. IR: Infrared Radiation; FLIR; MA-rFTIR: macroscopic reflection mode Fourier transform infrared.

\begin{tabular}{|c|c|c|c|}
\hline Test Sample & Method & Excitation Source & Camera \\
\hline Mondrian & IR-A reflectography & halogen & Goodrich \\
\hline Mondrian & IR-B reflectography & halogen & FLIR X6540sc $2.2 \mu \mathrm{m}$ filter \\
\hline Mondrian & IR-B+C' active thermography & halogen & FLIR X6540sc \\
\hline Mondrian & IR-C' active thermography & Xenon flash & FLIR Phoenix \\
\hline
\end{tabular}

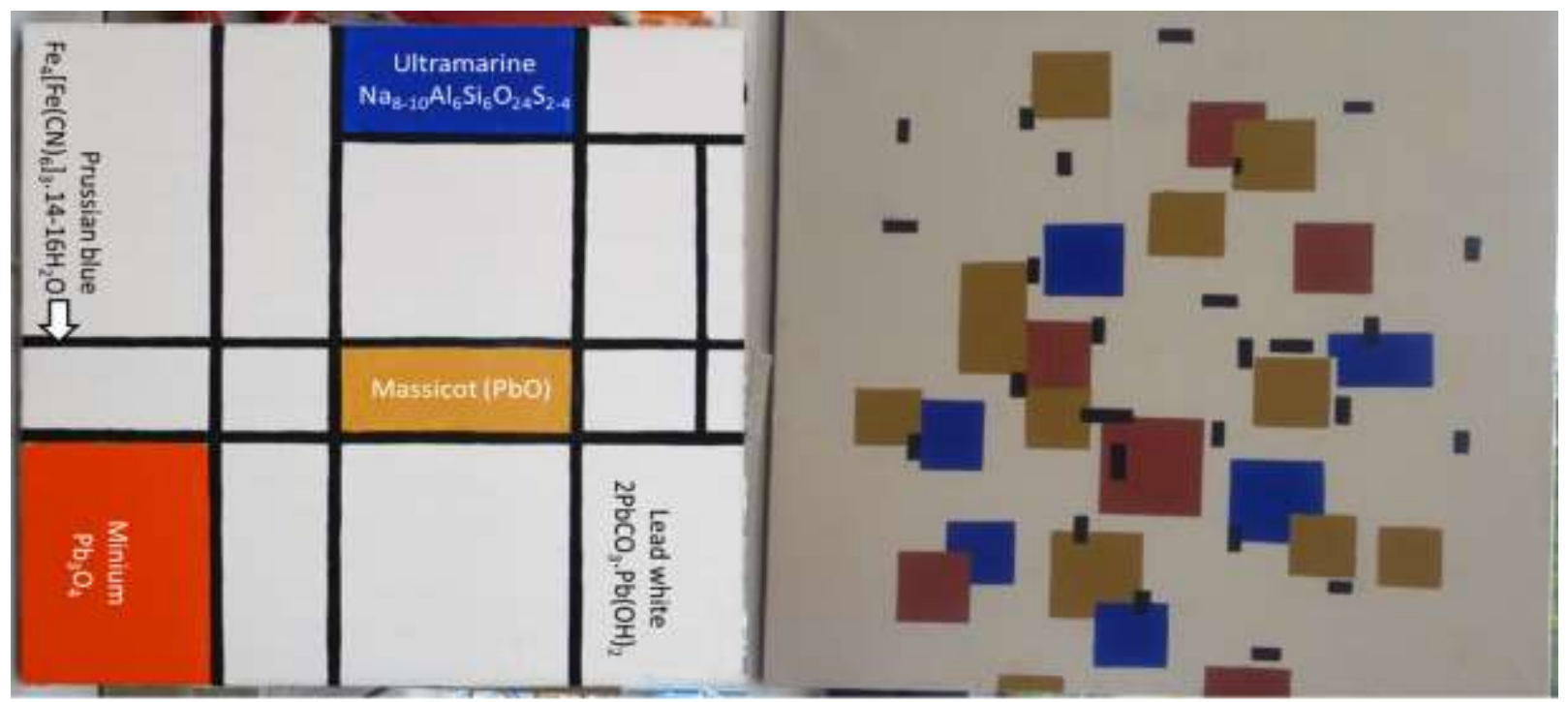

Composition no. 1

Composition no. 2

Fig. 1. Overview of the compositions Left: composition underneath, right: top layer composition. 


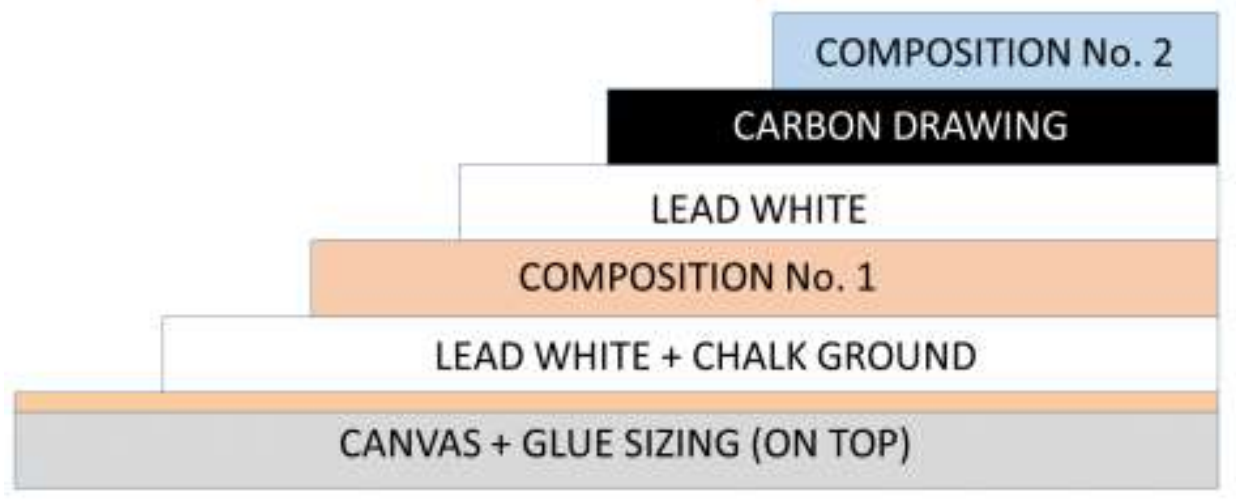

Fig. 2. Schematic overview of the different layer stacking of Mondrian painting.

\section{Results \& Discussion}

The IR-A results, shown in Fig. 3 are performed with halogen illumination where the prussian blue lines of the composition below the lead white cover layer appear. The massicot and ultramarine pigments appear only vague.

For the thermal inspection, the camera is used within the IR-C' spectrum. First, a halogen excitation source is used as active heat source directly aimed at the painting, exposing the painting to a sine wave excitation of $0.1 \mathrm{~Hz}$, blocking the thermal power using a double glass filter. The result is shown in Fig. 4 after Fourier transformation and phase analysis. The prussian blue lines are visible, but excessive post-processing is needed.

A second similar inspection is performed using pulsed thermography with a high power Xenon flash excitation of $5 \mathrm{~ms}$. The results, post-processed with PCA (bin 3) are presented in Fig. 5. It can be seen that not only the prussian blue lines but as well the massicot and ultramarine pigments could be clearly seen and the minium pigment could be vague distinguished. This can conclude that it is possible to evaluate the full sub-layered composition and that the results are similar or even better than the commonly used IR-A reflectography measurement.

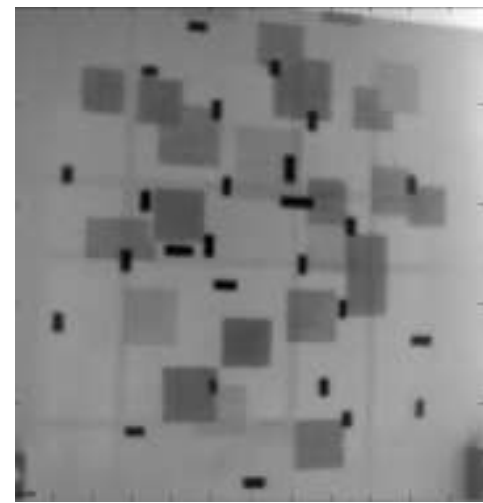

Fig. 3. IR-A results with Carbon illumination.

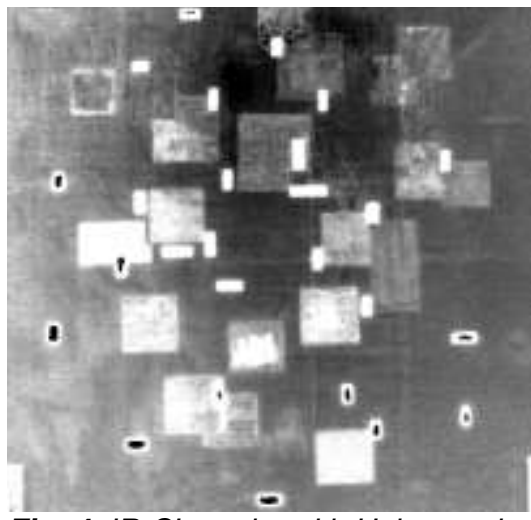

Fig. 4. IR-C' results with Halogen sine wave of $0.1 \mathrm{~Hz}$ after Fourier transform and phase analysis.

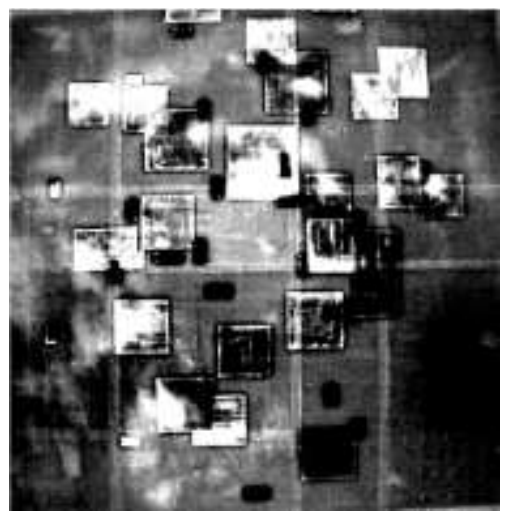

Fig. 5. IR-C' results with flash excitation after PCT analysis (bin 3).

With those results, we can conclude that the inspection of composition no. 1 is possible with a similar or even better accuracy using active thermography and an IR-B+C' camera with respect to the well-known IR-A reflectography technique. It should be marked that the selected test sample contains a very thick intermediate layer of lead white. Unfortunately, with state-of-the-art techniques, those paintings below a painting with a thick lead white layer are very difficult to inspect. With these results, we can conclude that the use of active thermography can provide a relatively fast way to evaluate the presence of sub-layer compositions.

\section{Conclusions}

The aim of this study is to compare the abilities of a broadband MWIR (IR-B+C') camera systems with the separate capabilities of a MA-rFTIR spectroscopy with respect to art work inspection. We inspected a Mondrian sample painting with different layers in order to perform the most known analyses. It is demonstrated that the underdrawings can be made visible when the lead white paint layers in between are not too thick. It is shown that the contrast is increased by performing active thermography analysis, with respect to the common IR-A reflectography when intermediate layers become thicker. From the results we can conclude that inspection of composition $n^{0} 1$ is possible with a similar or even 
better accuracy using active thermography and a IR-B+C' camera with respect to the well-known IR-A reflectography technique. It should be noticed that the selected test sample contains a very thick intermediate layer of lead white. Unfortunately, with state-of-the-art techniques, those paintings below a painting with thick lead white layer are very difficult to inspect. With these results we can conclude that the use of active thermography can provide a relatively fast way to evaluate the presence of sub-layer compositions.

\section{Acknowledgments}

This research was funded by the University of Antwerp and the Institute for the Promotion of Innovation by Science and Technology in Flanders (VLAIO) via support for the TETRA project, "SINT: Smart Integration of Numerical modeling and Thermal inspection," project number HBC.2017.0032. The researchers received funding from the Antwerp University IOF-council through project PSID-34924 entitled "Fast Broadband Lock-In Thermography for Fragile Structures Using System Identification."

\section{REFERENCES}

[1] C. Ibarra-Castanedo, M. Genest, J.-M. Piau, S. Guibert, A. Bendada, and X. P. V Maldague, "Active Infrared thermography Techniques For The Nondestructive Testing of Materials," in Ultrasonic and Advanced Methods for Nondestructive Testing and Material Characterization, WORLD SCIENTIFIC, 2007, pp. 325-348.

[2] G. Busse, "Techniques of Infrared Thermography: Part 4. Lock-in thermography," in Nondestructive Handbook, 3rd Editio., X. P. Maldague, Ed. ASNT Press: Columbus, OH, USA, 2001, p. 718.

[3] G. Busse, "Lockin-Thermography: Principles, NDE-applications, and trends," in Qirt 2014 Proceedings, 2014.

[4] C. Daffara, D. Ambrosini, R. Di Biase, R. Fontana, D. Paoletti, L. Pezzati, and S. Rossi, "Imaging data integration for painting diagnostics," 2009, vol. 7391, p. 73910X.

[5] J. K. Delaney, M. Thoury, J. G. Zeibel, P. Ricciardi, K. M. Morales, and K. A. Dooley, "Visible and infrared imaging spectroscopy of paintings and improved reflectography," Herit. Sci., vol. 4, no. 1, p. 6, 2016.

[6] J. Peeters, G. Steenackers, S. Sfarra, S. Legrand, C. Ibarra-Castanedo, K. Janssens, and G. Van der Snickt, "IR Reflectography and Active Thermography on Artworks: The Added Value of the 1.5-3 $\mu \mathrm{m}$ Band," Appl. Sci., vol. 8, no. 1, p. 50, Jan. 2018.

[7] C. Daffara, L. Pezzati, D. Ambrosini, D. Paoletti, R. Di Biase, P. I. Mariotti, and C. Frosinini, "Wide-band IR imaging in the NIR-MIR-FIR regions for in situ analysis of frescoes," O3A Opt. Arts, Archit. Archaeol. III, vol. 8084, pp. 808406-808406-12, 2011.

[8] D. Gavrilov, E. Maeva, O. Grube, I. Vodyanoy, and R. Maev, "Experimental comparative study of the applicability of infrared techniques for non-destructive evaluation of paintings," J. Am. Inst. Conserv., vol. 52, no. 1, pp. 4860, 2013.

[9] S. Sfarra, P. Theodorakeas, N. P. Avdelidis, and M. Koui, "Thermographic, ultrasonic and optical methods: A new dimension in veneered wood diagnostics," Russ. J. Nondestruct. Test., vol. 49, no. 4, pp. 234-250, 2013.

[10] D. Gavrilov, R. G. Maev, and D. P. Almond, "A review of imaging methods in analysis of works of art: thermographic imaging method in art analysis," Can. J. Phys., vol. 92, no. 4, pp. 341-364, 2014.

[11] W. Y. Jeong, C. J. Earls, W. D. Philpot, and A. T. Zehnder, "Inverse thermographic characterization of optically unresolvable through cracks in thin metal plates," Mech. Syst. Signal Process., vol. 27, pp. 634-650, Feb. 2012.

[12] G. Steenackers, J. Peeters, S. Verspeek, and B. Ribbens, "From Thermal Inspection to Updating a Numerical Model of a Race Bicycle: Comparison with Structural Dynamics Approach," Appl. Sci., vol. 8, no. 2, Feb. 2018. 